\title{
Desarrollo rural y envejecimiento: caso de estudio municipio de Chinavita, Boyacá, Colombia
}

\author{
Rural development and aging: case study in Chinavita, Boyacá, Colombia \\ Érica Paola López' \\ Lina Marcela Martínez ${ }^{2}$ \\ Camilo Andrés Martínez-Cañas ${ }^{3}$ \\ Amanda Vargas-Prieto ${ }^{4}$
}

Recibido: junio 20 de 2017

Aceptado: noviembre 04 de 2017

\begin{abstract}
Resumen
El objetivo de esta investigación es determinar las implicaciones del envejecimiento en el desarrollo del departamento de Boyacá, analizando el periodo entre 1985 y 2005, como datos históricos, y del 2006 al 2020 como datos estimados. Se recurrió a un estudio de caso realizado en el municipio de Chinavita, Boyacá, Colombia. Los resultados permitieron establecer que existe una relación directa entre el crecimiento económico y los procesos migratorios de la población más joven, lo que impacta directamente en la capacidad productiva del municipio. Algunos de los factores que causan los altos niveles de migración son la falta de oportunidades de empleo y educación de las zonas rurales. A esto se suman posibles riesgos en la sostenibilidad tanto del sistema de seguridad y protección social como de las finanzas públicas, destinadas a programas de atención del adulto mayor. La ausencia de empleo formal, que permita que los jóvenes coticen en el sistema de seguridad y protección social, pone de manifiesto una posible crisis en el sistema pensional. Lo anterior evidencia la necesidad de una política de Estado seria y responsable, que aborde el tema del envejecimiento de manera integral.
\end{abstract}

Palabras clave: envejecimiento, desarrollo rural, crecimiento económico, seguridad social, migración.

\begin{abstract}
The objective of this research is to determine the implications of aging of people on the development of Boyacá State, analyzing the period between 1985 and 2005 as historical data and from 2006 to 2020 as estimated data. For this purpose, we used a case study carried out in the municipality of Chinavita, province of Valle de Tenza in Boyacá, Colombia. The application of the methodology allowed determining that there is a direct relationship between economic growth and the migration processes of the younger population, which directly impacts on the profitable capability of the municipality. In this sense, some of the most important factors that cause high levels of migration are the lack of opportunities for employment and education opportunities in rural areas. In addition to this, potential risks in the sustainability of both the social security and protection system and besides, public finances aimed to elderly care programs. The absence of formal employment, which allows young people to contribute to the social security and protection system, reveals a possible crisis in the pension system. In this context, it is shown the need for a serious and responsible state policy that addresses the issue of aging of people in a comprehensive manner.
\end{abstract}

Keywords: aging of people, rural development, economic growth, social security, migration.

1 Economista, Magíster en Estudios y Gestión del Desarrollo, Independiente, Bogotá, Colombia. E-mail: eriklop12_30@hotmail.com

2 Profesional en Relaciones Internacionales y Estudios Políticos, Magíster en Estudios y Gestión del Desarrollo, Servicio Jesuita a Refugiados, Bogotá, Colombia. E-mail: Imartinezguti88@gmail.com

3 Profesional en Gobierno y Relaciones Internacionales, Magíster en Estudios y Gestión del Desarrollo, Revista Semana, Bogotá, Colombia. E-mail: camilomartinezca@gmail.com

4 Administradora de Empresas, Doctora en Ciencias Económicas, Universidad de la Salle, Bogotá, Colombia. E-mail: amvargas@unisalle. edu.co 


\section{Introducción}

Según la Organización Mundial de la Salud, OMS, (2015), en los últimos años la proporción de personas mayores de 60 años ha venido aumentando más rápidamente que cualquier otro grupo de edad en casi todos los países. Hechos como el aumento de la esperanza de vida y la disminución de la tasa de fecundidad, son las causas principales de este fenómeno, que, si bien representa una mejor calidad de vida de las personas, también implica grandes retos para los Estados. La Comisión Económica para América Latina y el Caribe, CEPAL, ha identificado dos características del proceso de envejecimiento en la región. En primer lugar, el envejecimiento se produce y se seguirá produciendo a un ritmo más rápido que el registrado históricamente. En segundo, se dará en un contexto caracterizado por una alta incidencia de pobreza, una persistente y aguda inequidad social, un escaso desarrollo institucional, una baja cobertura de la seguridad social y una probable tendencia hacia la disminución del apoyo social, (CEPAL, 2004).

En Colombia, en los últimos años se ha venido registrando un mayor crecimiento del número de personas con 60 años o más, reflejado en que para 1985 esta población representaba solo el 7\% del total de la población, cifra que aumentó al $10 \%$ en el 2014 y que llegará al 23\% para el 2050. Según un estudio realizado entre Fedesarrollo y la Fundación Saldarriaga, (2015), los cambios en la estructura etaria de la población colombiana difieren entre áreas urbanas y rurales. Hechos como la migración rural-urbana selectiva por edad y sexo, una mayor mortalidad en las zonas rurales y el proceso de envejecimiento de los adultos que migraron hacia las ciudades en años anteriores, hace que la población de 60 años o más tienda a crecer de manera más que proporcional en las zonas urbanas frente a las rurales. Sin embargo, el proceso migratorio del campo a la ciudad ha aumentado significativamente el número de hogares rurales conformados exclusivamente por personas mayores, pasando de un $2.8 \%$ en
1990 a un $5.7 \%$ en 2010 . Al revisar los procesos de envejecimiento a nivel territorial, se puede observar que Boyacá es el de mayor peso relativo con un $12,4 \%$, tres puntos porcentuales más que el promedio nacional. A esto se suma que, según el Censo Nacional Agropecuario, (2014), Boyacá es el departamento que cuenta con el mayor número de campesinos que superan los 60 años.

Teniendo en cuenta lo anterior, la presente investigación busca determinar cuáles son las implicaciones del envejecimiento en el desarrollo rural del departamento de Boyacá. Se plantean los siguientes objetivos específicos: identificar los determinantes del proceso de envejecimiento del sector rural en el departamento; establecer la relación entre envejecimiento y desarrollo rural; y determinar las implicaciones del envejecimiento en el desarrollo rural de Boyacá. El trabajo pretende aportar a los estudios sobre desarrollo rural, especialmente cuando la transformación del campo es uno de los principales retos del país en el post-acuerdo.

Los objetivos se alcanzaron a través de un estudio de caso realizado en el municipio de Chinavita, Boyacá, analizando el periodo entre 1985 y 2005, como datos históricos, y del 2006 al 2020 como datos estimados. El documento inicia con la presente introducción, donde se evidencia la necesidad de estudiar el envejecimiento y sus implicaciones en el desarrollo rural de Boyacá. Luego se detallan la metodología y las técnicas de recolección de información. En tercer lugar se presentan los resultados, para finalizar con las conclusiones.

\section{Metodología}

\subsection{Estudio de caso}

La investigación utilizó la metodología cualitativa del tipo estudio de caso, con el fin de identificar patrones y configuraciones entre el envejecimiento y el desarrollo rural, ya que esta relación requiere una descripción, interpretación y explicación 
detallada de las implicaciones de la primera sobre la última. Stake, (1995), sostiene que el estudio de caso no es la elección de un método sino más bien la elección de un objeto a ser estudiado. En tanto enfoque de investigación, un estudio de caso es definido por el interés en casos individuales antes que por los métodos de investigación utilizados.

En este sentido, el estudio de caso permitió analizar el envejecimiento y el desarrollo rural en Chinavita, tomando como base el periodo entre 1985 y 2005, como datos históricos, y del 2006 al 2020 como datos estimados, según proyecciones del Departamento Administrativo Nacional de Estadística, DANE. Lo anterior gracias a que este método tiende a focalizar en un número limitado de hechos y situaciones, para poder abordarlos con la profundidad requerida para su comprensión holística y contextual, (Vasilachis-de Gialdino, 2006).

\subsection{Municipio de Chinavita}

Chinavita es un municipio de la provincia del Neira en el suroriente de Boyacá, situado a $75.2 \mathrm{~km}$ de la ciudad de Tunja. Su extensión territorial es de $128 \mathrm{~km}^{2}$, lo que representa el $0.6 \%$ del área total del departamento. La densidad poblacional es de 28 habitantes por kilómetro cuadrado, en donde el $65 \%$ vive en zona rural. Su población total es de 3.499 personas, de las cuales el $50 \%$ son hombres y el 50\% mujeres; de ellos, aproximadamente el $24 \%$ son personas mayores de 60 años, lo que convierte al municipio en el número 12 de 123, con mayor índice de envejecimiento en Boyacá. De los 608 adultos mayores que existen en el municipio, el $96.3 \%$ pertenece al programa Colombia Mayor, (DANE, 2005).

Al ser un municipio mayoritariamente rural su economía gira en torno a la producción agropecuaria, lo que representa un 19\% del producto interno bruto local. La mayoría de sus habitantes se dedican al cultivo de la papa, caña de azúcar, tomate, hortalizas, plátano, café, frutas y fique, en su mayoría como pancoger. Para estas labores se usan métodos rudimentarios como el arado, chuzo y bueyes, lo que demuestra los bajos niveles de tecnificación del campo. En ganadería, predomina el ganado vacuno con pequeña presencia de porcinos, caprinos, equinos y aves de corral, que suplen las necesidades diarias de las personas. Lo anterior como consecuencia de los bajos niveles de producción agropecuaria, que se agudizan por la ausencia de asistencia técnica, la falta de maquinaria, insuficientes canales de comercialización y bajos niveles de asociatividad, problemas comunes a otros municipios del departamento y el país (Sierra-Roberto, 2012; López-Posada, \& Pachón-Ariza, 2017).

Según el Departamento Nacional de Planeación, DNP, Chinavita presenta una tipología municipal D y un entorno de desarrollo intermedio. Esto se refleja en: una importante afinidad ambiental, excelentes niveles de seguridad y un buen desempeño institucional. Sin embargo, en la dimensión económica y de calidad de vida, el municipio cuenta con un Índice de Necesidades Básicas Insatisfechas, INBI, del 35.79\%, por encima del promedio nacional de $19.66 \%$ (DANE, 2005). El Índice de Pobreza Multidimensional, IPM, es del $64.4 \%$, donde para la zona rural aumenta al $75.8 \%$. En el IPM las variables con mayor grado de incidencia son: empleo informal $96.61 \%$; bajo logro educativo 87.03\%; y alta tasa de dependencia económica $57.3 \%$. Al bajo logro educativo se suma un nivel de analfabetismo del $14.8 \%$ y una tasa de mortalidad infantil del $20.8 \%$, ambas por encima del promedio departamental.

\subsection{Técnicas de recolección de información}

Teniendo en cuenta el enfoque de la triangulación, el cual se refiere al uso de varios métodos (tanto cuantitativos como cualitativos), de fuentes de datos, de teorías, de investigadores o de ambientes en el estudio de un fenómeno, se recurrió a diferentes técnicas de recolección de datos, como: entrevistas semiestructuradas, entrevistas a expertos y la estadística descriptiva. 


\section{Entrevista semiestructurada}

Estas entrevistas se basan en una guía de asuntos o preguntas y el entrevistador tiene la libertad de introducir preguntas adicionales para precisar conceptos u obtener mayor información sobre los temas deseados; es decir, no todas las preguntas están predeterminadas (Sampieri, Fernández \& Lucio, 2006). Esta técnica permitió acceder a información propia del territorio y sus elementos constitutivos, como: cultura, datos históricos y del contexto, dinámicas económicas, sociales y poblacionales y características particulares de la ubicación. Es decir, se obtienen datos que solo surgen de la experiencia de quien ha vivido en el territorio y ha sido partícipe de los procesos de desarrollo allí ocurridos. Se pueden distinguir varios tipos de entrevista semiestructurada, de los cuales se aplicaron: la entrevista focalizada, entrevista a expertos y entrevista no estructurada o abierta.

Entrevista focalizada: consiste en reuniones de grupos pequeños o medianos, 3 a 10 personas, en las cuales los participantes conversan en torno a uno o varios temas en un ambiente relajado e informal, bajo la conducción de un especialista en dinámicas grupales (Sampieri, Fernández, \& Lucio, 2006). En este caso el grupo focal contó con la presencia de 9 líderes sociales del municipio, con edades entre 45 y 71 años, con quienes se construyó una línea de tiempo con eventos significativos para la población. La selección de los participantes se hizo teniendo en cuenta características como: nacieron o viven hace más de 3 años en el municipio; gran parte de su vida la han dedicado a actividades agropecuarias; ellos o sus hijos han migrado de forma temporal o permanente a las ciudades en busca de oportunidades laborales o educativas; tienen a su cargo personas aún mayores, hijos y nietos o viven solos, ya que sus hijos se radicaron en otras ciudades; existe presencia de hombres y mujeres en similar proporción; han sido líderes históricos en la comunidad; una vez alcanzaron su pensión deciden irse a vivir al campo; y en los últimos años han trabajado por la recuperación de prácticas tradicionales agrícolas dentro del municipio.

Entrevista a expertos: Meuser y Nagel, (1991), analizan las entrevistas a expertos como una forma específica de aplicar entrevistas semiestructuradas. El experto se integra en el estudio, no como un caso individual, sino como una representación de un grupo de expertos específicos. La guía de entrevista se estructuró en tres partes: la primera enfocada en explorar la concepción de desarrollo rural, la segunda el concepto de envejecimiento, y la tercera en establecer la relación entre ambas variables. Esta técnica se aplicó a Rafael Mejía y Cecilia López Montaño, ambos expertos de la Misión Rural y grandes conocedores de los temas rurales en el país, con el fin de fortalecer las bases teóricas no solo sobre los temas de desarrollo rural y envejecimiento de forma aislada, sino también con el fin de dar indicios sobre el tipo de relación que existe entre ambas variables y su nivel de incidencia.

Entrevista no estructurada o abierta: las preguntas de este tipo de entrevista pueden ser de carácter abierto y flexible. Existe una interacción entre el entrevistador y el entrevistado, el cual está vinculado por una relación de persona a persona cuyo deseo es entender más que explicar. Este tipo de entrevista se aplicó a la profesional encargada de la Entidad Prestadora de Servicios de Asistencia Técnica, Epsagro, del municipio de Chinavita. También se entrevistó a la responsable de la implementación de la política social de protección al adulto mayor por parte de la alcaldía del municipio.

\section{Estadística descriptiva}

La estadística descriptiva sirvió para realizar el análisis de las siguientes variables: asignación presupuestal del sector agropecuario entre 2003 y 2014, datos anuales según el DNP; causas de los procesos migratorios en Chinavita en el año 2000; saldos netos migratorios por género en Boyacá; índice de envejecimiento por género en Boyacá y 
Chinavita; crecimiento del Producto Interno bruto, PIB, en Chinavita de 2011 al 2015, datos anuales según el DNP; producción por semestre de trigo y maíz en Boyacá del 2005 al 2016, datos anuales según Federación Nacional de cultivadores de cereales y leguminosas; participación de la población de Chinavita en el fondo de pensiones 2005; proyección del costo de la población no afiliada al fondo de pensiones en Chinavita para 2017, según datos de 2005; y estimado presupuestal de gastos estampilla pro anciano: 2012-2015, datos del Marco Fiscal de Mediano Plazo, MFMP, de Chinavita (DNP, 2010).

\section{Resultados y discusión}

3.1 Procesos migratorios como consecuencia de los modelos de desarrollo

Según la Misión para la Transformación del Campo (DNP, 2015), el 84,7\% del territorio nacional posee características de ruralidad. Allí, vive el 30,4\% del total de la población colombiana. Pese a esto, no existe una política de Estado que promueva el desarrollo rural entendido más allá de lo agropecuario. Como consecuencia directa, son importantes los procesos migratorios que se han venido dando de lo rural a lo urbano, y que para el caso concreto de Chinavita, se convierte en uno de los desencadenantes del envejecimiento en el municipio. A continuación se analizan las principales causas de la migración, para luego establecer su relación con los procesos de envejecimiento.

Principales causas de los procesos migratorios en Chinavita

En la entrevista con Rafael Mejía, él menciona que no existe un enfoque de desarrollo rural en la política pública en Colombia. Dicha afirmación, es corroborada por los campesinos entrevistados quienes manifiestan que "no hay una legislación apropiada para ellos". De ahí que, el campo sigue siendo un simple proveedor de mano de obra y recursos naturales para los procesos industriales de la ciudad (Kay, 2007). Lo anterior, en contraste con las propuestas de alternatividad al desarrollo pensadas desde los territorios, que, como lo establecieron Pérez-Correa y Farah-Quijano (2002), pueden convertirse en una posible solución a las problemáticas urbanas del país.

Lo anterior evidencia la marginación del sector rural en la asignación presupuestal de los distintos Gobiernos. Según la MTC, (2015), en los últimos 23 años, el presupuesto del sector agropecuario pasó de $\$ 896$ millones en 1990 a $\$ 3.4$ billones en el año 2014. Sin embargo, su comportamiento ha sido muy inestable, especialmente entre el año 2000 y 2005 cuando se alcanzó su punto más bajo. Estos recursos representan una asignación promedio del $6 \%$ del PIB, en donde desde mediados de la década de 1990 hasta el año 2003, la participación registró una caída importante, pasando del 10\% en 1990 a poco más del 3\% en 2003. Desde esta fecha hasta el 2014, la participación porcentual del sector ha sido de las más bajas, especialmente si se compara con los recursos destinados a defensa y seguridad. En este sentido, sorprende la baja destinación hecha a educación, que para los años señalados tiene una asignación promedio de $4 \%$ del PIB, lo cual va en contravía de su importancia en lo que a desarrollo rural se refiere.

La marginación del sector se ve directamente reflejada en los entes territoriales. En el caso específico de Chinavita la asignación presupuestal para lo rural no es clara. Sin embargo, al revisar el Plan de Desarrollo: Unidos por Chinavita, (2016-2019), lo que se observa es que no existen programas $y / 0$ proyectos específicos para promover el sector rural en el municipio (Alcaldía de Chinavita, 2016).

En este contexto, el sentir del campesino es que los pocos recursos que existen, son destinados a capacitaciones que no se materializan en ayudas directas para la implementación de buenas prácticas en los procesos productivos: "(...) aquí llegan muchos recursos, pero eso solo se van en capacitaciones (...) En todo este tiempo que 
llevamos (en la asociación de ganaderos), casi más de tres años, no nos han ayudado para un purgante de un becerro que son mil pesos. A esto se suma que la entrega de los pocos insumos que se dan, se hace de manera discrecional, en su mayoría, bajo un componente político, generando importantes niveles de desconfianza y falta de credibilidad hacia la institucionalidad.

Por otro lado, para Rafael Mejía "la oferta de bienes públicos como vivienda, salud y educación, se ha concentrado en las ciudades y no en el campo". Del total de los campesinos que participaron del grupo focal, el 57\% manifestaron haber migrado a la ciudad siendo niños y/o jóvenes en busca de oportunidades de empleo y educación. Al igual que ellos, la mayoría de sus hijos han salido del municipio por las mismas razones: "De mis 8 hijos, 6 están viviendo en Bogotá. Otro está en Guaduas (y la más pequeña) está por allá trabajando en San Luis". Si estos testimonios se contrastan con cifras del último censo nacional (DANE, 2005), lo que se puede encontrar es que del total de los habitantes que declararon haber salido de Chinavita en el año 2000 , el $33 \%$ lo hicieron por asuntos familiares, el $29 \%$ por dificultad para conseguir trabajo y el $9 \%$ por necesidad de educación, ver figura 1.

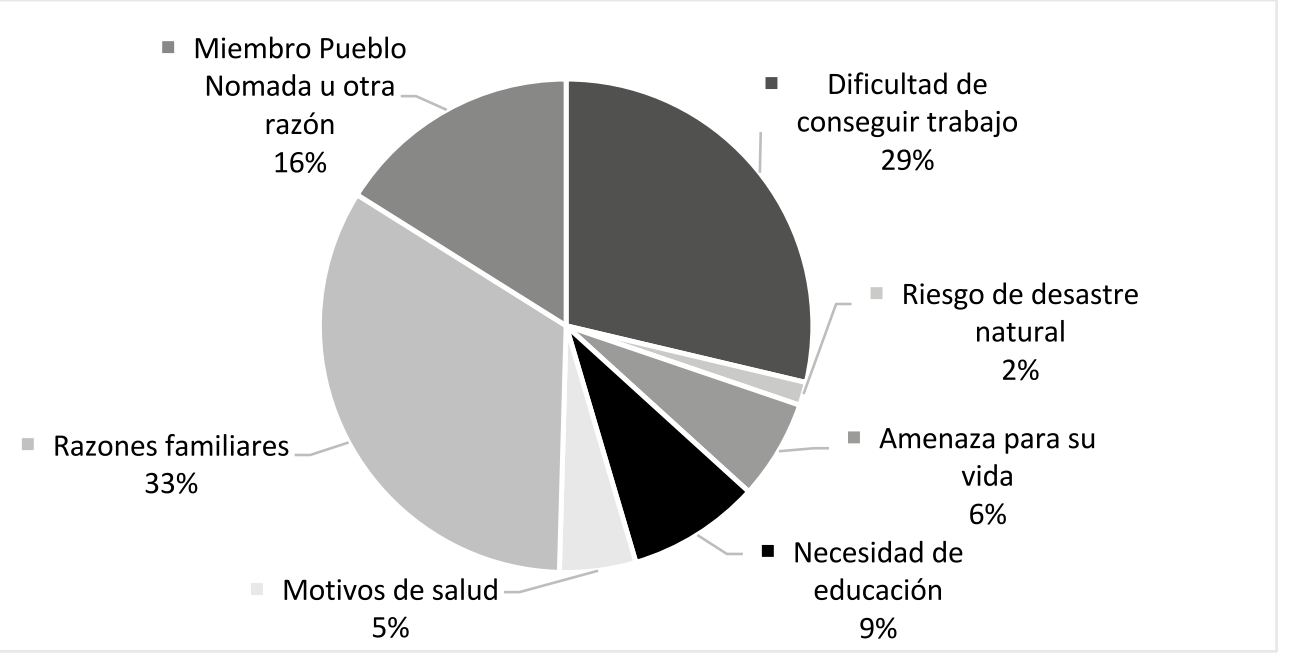

Figura 1. Causas de la migración de Chinavita a otros departamentos en el 2000, datos del DANE (2005).

Lo anterior corresponde a las consecuencias directas de la implementación de políticas bajo el enfoque estructuralista, el cual trajo consigo la agudización de la pobreza en el sector rural (Pérez-Correa, \& Farah-Quijano, 2002). Si se revisa el Índice de Pobreza Multidimensional, IPM, se observa que para la zona rural de Chinavita existe un IPM del $75,8 \%$, casi el doble del urbano, $39.8 \%$. Los principales niveles de pobreza se reflejan en las variables asociadas a: empleo informal $96.61 \%$ ), bajo logro educativo $87.03 \%$, y alta dependencia económica 53.7\%. En 2005, del total de la población registrada el $16 \%$ no sabían leer ni escribir, de los cuales el 49\% tenían 60 años o más. A esto se suma que solo el $4 \%$ habían logrado terminar una carrera profesional y/o postgrado (DANE, 2005).

En este contexto, para Rafael Mejía y la representante del Epsagro, la educación básica que se ha brindado en el sector rural, lejos de favorecer el desarrollo del campo ha incidido en la migración de la población más joven hacia las ciudades. Lo anterior tiene una relación directa con la pérdida del arraigo por la tierra, lo cual fue consecuencia directa de las políticas modernistas que intentaron dejar atrás prácticas y tradiciones rurales consideradas obstáculos en los procesos de 
modernización. Para los campesinos entrevistados, es normal que los jóvenes busquen un proyecto de vida alejados del campo, en la medida en que socialmente se ha construido el concepto de que para ser "alguien en la vida" es necesario migrar a la ciudad.

\section{Relación entre migración y envejecimiento}

Según el estudio de proyección nacional y departamental de población 2005-2020 (DANE, 2010), pese a que el Saldo Neto Migratorio, SNM, en el departamento de Boyacá ha sido históricamente negativo, se prevé que para los últimos años éste comience a descender en la medida en que la población boyacense está migrando hacia sus propios cascos urbanos: Tunja, Duitama, Sogamoso y Chiquinquirá. Dicha situación se corrobora en las figuras 2 y 3 , que muestran una desaceleración de los procesos migratorios.

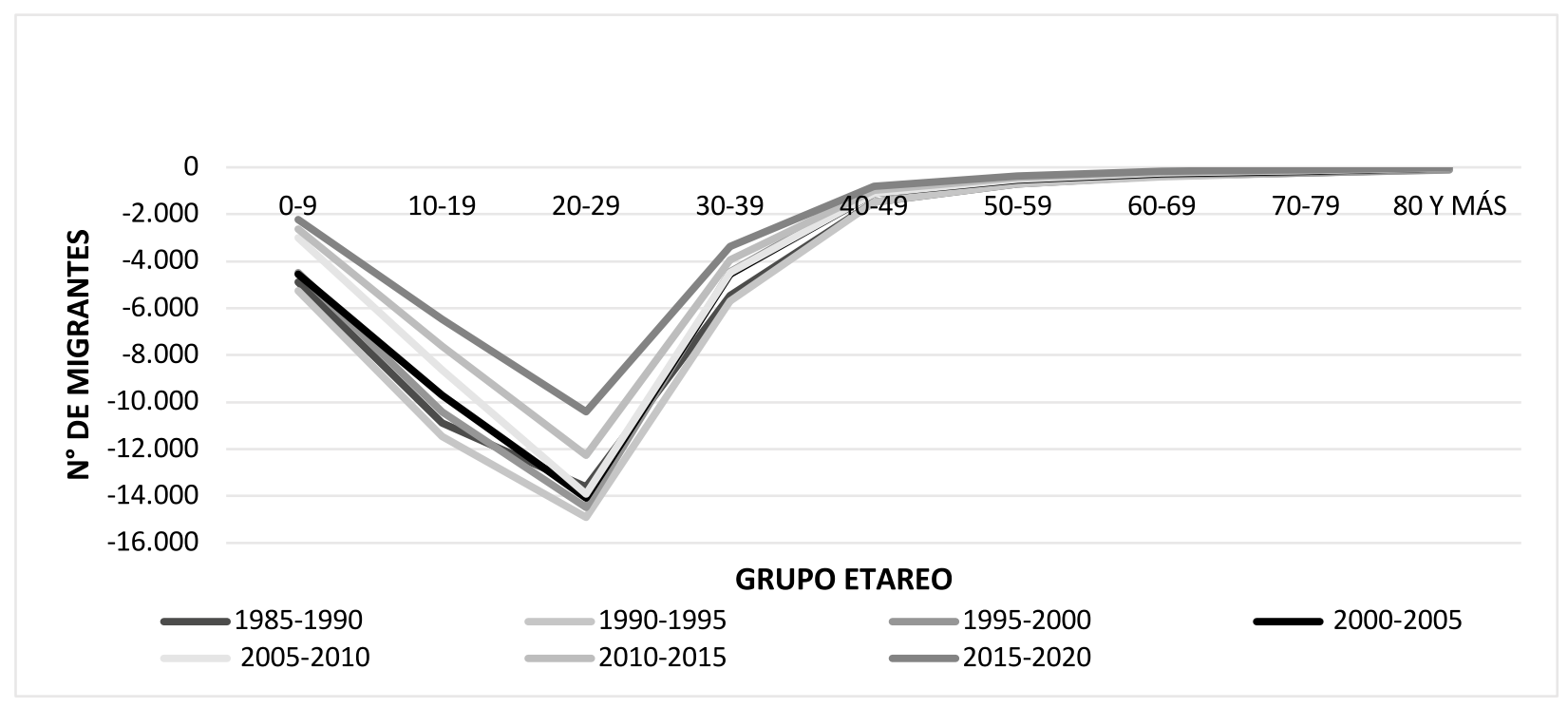

Figura 2. Saldo neto migratorio para hombres en Boyacá (DANE2005).

Como se observa en la figura 2, existe una disminución significativa del SNM para el periodo 2015-2020, especialmente para los hombres entre los 10 y 19 años. A esto se suma un mayor nivel de migración para los hombres entre los 20 y 29 años, especialmente en el periodo comprendido entre 1990 y 1995; adicionalmente, son claros los bajos niveles de migración para las personas entre los 50 y 80 años o más. 


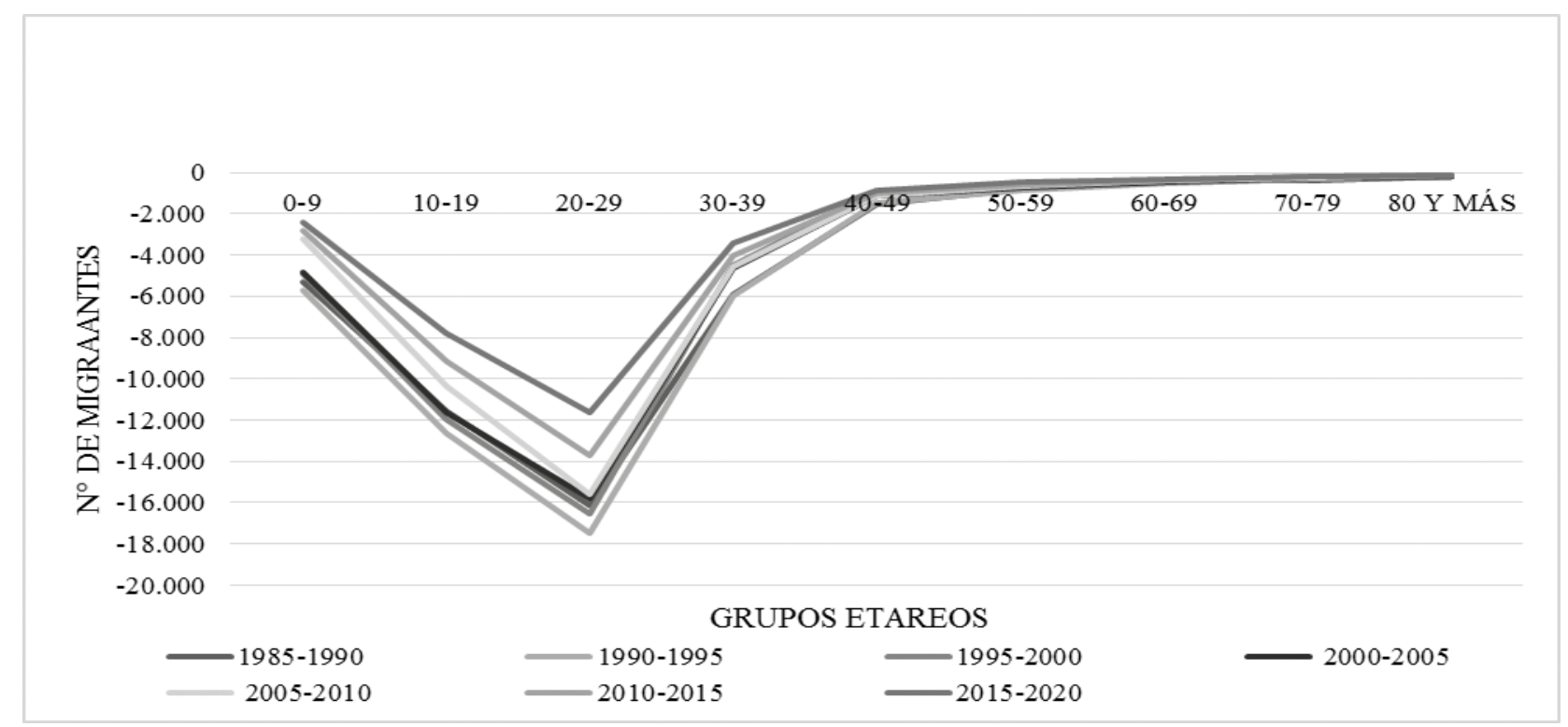

Figura 3. Saldo neto migratorio para mujeres en Boyacá (DANE, 2005).

La figura 3 permite observar que existe una mayor tendencia de migración en las mujeres entre los 20 y 29 años, seguido por quienes tienen 10 a 19 años. Al igual que en los hombres, existe una disminución del SNM para el periodo 2015-2020. Sin embargo, en el caso de las mujeres, quienes dejan de migrar en mayor proporción son quienes tienen entre 20 y 29 años. Finalmente, se repiten los mismos bajos niveles de migración de la población entre los 50 y 80 años o más, en donde entre los años de 1990 y 1995 se presentan las mayores migraciones para estos grupos etarios.

Pese a la disminución de los SNM, el departamento sigue enfrentando importantes procesos migratorios del campo a la ciudad que inciden directamente en los índices de envejecimiento del sector rural. Si bien las tendencias muestran que se ha reducido la migración a otros departamentos, especialmente Cundinamarca y Bogotá, la salida de población campesina a los cascos urbanos refleja un cambio en la vocación agrícola del territorio. Para el caso específico de Chinavita, un municipio mayoritariamente rural, 460 personas respondieron positivamente a la pregunta sobre si había migrado en los últimos 5 años respecto a su lugar de nacimiento durante la aplicación del Censo 2005. Es decir, un 13\% del total de la población que vivía allí para ese momento (DANE, 2005). El $49 \%$ de estas personas migraron a zonas urbanas de Boyacá, seguido de un 32\% y $9 \%$ a Bogotá y otros municipios de Cundinamarca, respectivamente. Del total de los migrantes, el $26 \%$ correspondían a personas entre 10 y 19 años, el $21 \%$ entre 20 y 29 años y el $16 \%$ entre 30 y 39 años. Por lo tanto, se corrobora que, según los datos disponibles, quien mayoritariamente migra a las ciudades es la población más joven.

Para la encargada de la implementación del programa de adulto mayor en el municipio, esta situación se presenta porque:"(a los) jóvenes, desde la misma familia y las instituciones educativas, no se les educa para que sigan en la tierra. Entonces, terminan el bachillerato, se van a estudiar o buscar trabajo donde sea". En esto coinciden el grupo de campesinos entrevistados, cuando mencionan que en la actualidad los jóvenes prefieren migrar a las ciudades a estudiar carreras distintas al sector. En este contexto, es válido pensar en la incorporación de nuevas tecnologías que hagan más atractivo el campo para estos grupos etarios. 
Lo anterior, en línea directa con los principales postulados neoestructuralistas, en donde se propugna por una política agraria diferenciada en favor de los campesinos que les permita entrar al mercado desde la incorporación de nuevas tecnologías (CEPAL, 1990).

Al ser la población más joven quienes migran a las zonas urbanas en busca de oportunidades laborales y de educación, se observa un envejecimiento poblacional; si se revisa el índice de envejecimiento en Chinavita, se puede observar cómo para 1985 éste se encontraba en un $22 \%$ mientras para el 2020 llegará, según aproximaciones, a un 59\%. Al compararse con el índice a nivel departamental, para los mismos periodos la cifra corresponde a un $14 \%$ y un $43 \%$, respectivamente. Lo anterior, con un efecto directo en los niveles de crecimiento económico de Chinavita, de lo cual se hablará más adelante.

3.2 Impacto en la reducción de la economía del territorio

En términos económicos, se encontró que el envejecimiento impacta la economía del territorio, en primer lugar, mediante la pérdida de mano de obra productiva que sea capaz de sostener el desarrollo rural, y posteriormente, esto se ve reflejado en la disminución del crecimiento económico y el detrimento de la productividad.

\section{Disminución del crecimiento económico}

El envejecimiento incide directamente, y de forma negativa, en las economías de los territorios. Para la Cepal (2000), el principal desafío se proyecta en términos económicos a través de factores como el crecimiento, el ahorro y la inversión y la oferta de mano de obra. Adicionalmente, Andrews, (2000) identifica que el envejecimiento de la población destruye la competitividad de los territorios, perpetuando y sometiendo a la falta de crecimiento y de la contracción de los recursos económicos. De esta manera, la Cepal y Andrews, (2000) plantean como implicación recurrente del envejecimiento sobre el desarrollo rural, las afectaciones del crecimiento económico mediante la disminución de población económicamente activa que sea capaz de mantener las estructuras productivas de las economías locales.

En esta línea, las entrevistas focalizadas realizadas a la comunidad y a expertos, permiten confirmar que el crecimiento económico se ve directamente afectado por el envejecimiento. En el grupo focal con los campesinos se corroboró que la mano de obra de Chinavita se ha ido perdiendo, situación que ha impactado negativamente en las fuentes de ingreso del núcleo familiar que dependen casi exclusivamente de estas entradas para cubrir sus necesidades básicas. Los campesinos y dueños de las tierras fueron enfáticos al señalar que su ingreso se ha visto seriamente afectado por el alto nivel de envejecimiento por el que ha atravesado el municipio, pues, al no haber mano de obra que trabaje la tierra, no hay como generar mayores ingresos. Igualmente, el campesinado manifiesta que el ahorro es otra de las variables afectadas, ya que, al hacerse más costosos los requerimientos de tiempo para trabajar la tierra y una mano de obra cada vez más costosa como producto de su escasez, los términos de intercambio les favorecen cada vez menos, y esto a su vez reduce los recursos para el ahorro. Además, esta práctica en entidades bancarias no es una opción que genera confianza en la comunidad, pues los costos bancarios terminan acabando con su capital y sus bajos niveles de ahorros posibles.

En relación a los expertos consultados, coinciden en señalar que cuando la gente se está envejeciendo se acaba con el bono demográfico y no hay población económicamente activa para sostener las economías de los territorios, lo que impacta directamente sobre el crecimiento económico. En este sentido, Rafael Mejía sostuvo que: "un envejecimiento, al nivel al que ha llegado Boyacá, tiene una repercusión directa sobre su crecimiento económico, pues no hay personas dispuestas a quedarse en el campo y a sostener la producción, impactando las economías locales 
que ya no tendrán cómo crecer". Esta afirmación encierra la implicación económica por excelencia, pues el crecimiento económico, como condición necesaria (aunque no exclusiva) para alcanzar el desarrollo rural no será alcanzable como resultado del envejecimiento crítico que ha alcanzado tanto Chinavita como Boyacá.
En la figura 4 se observa que el PIB de Chinavita se ha visto impactado de manera negativa, ya que, según datos del DANE, a nivel de municipio, el crecimiento, en los últimos años, ha sido del $0 \%$, estancamiento que se explica ante la inexistencia de una población económicamente activa que pueda sustentar algún tipo de crecimiento económico.

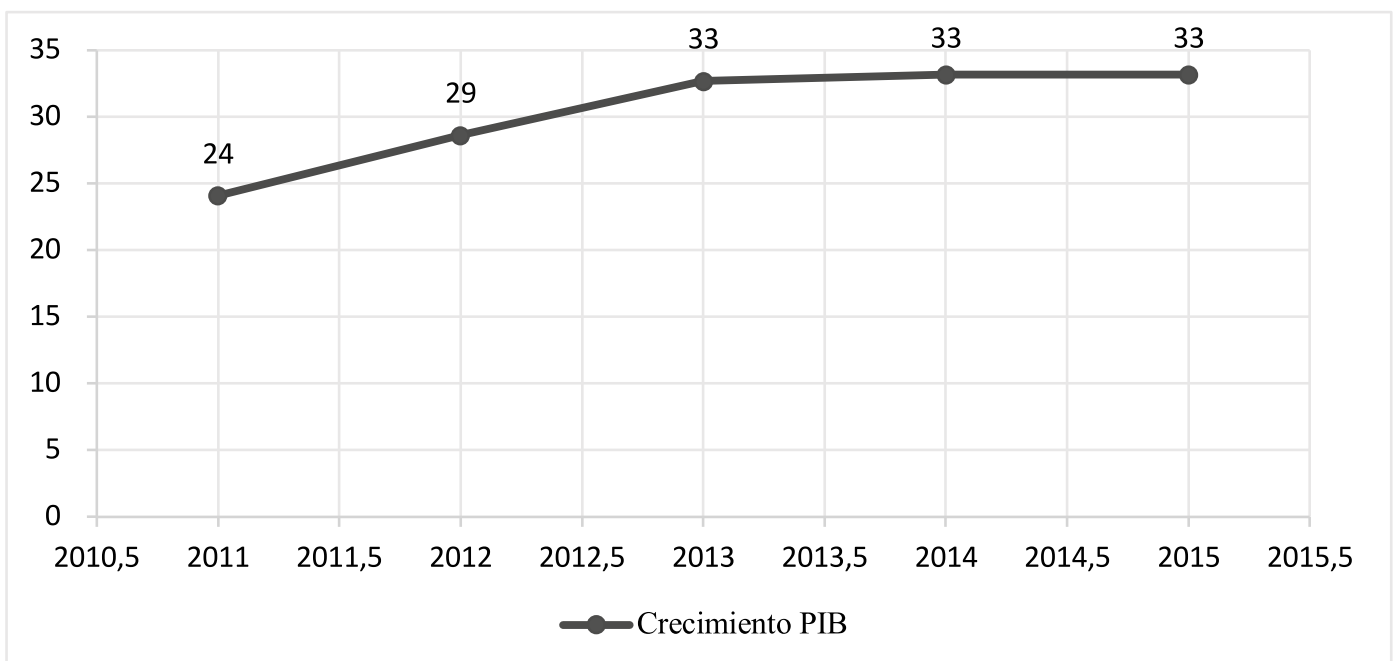

Figura 4. Crecimiento del PIB de Chinavita, a partir de datos del DANE (2005).

Lo anterior permite establecer que una de las principales implicaciones del envejecimiento se da en términos de la disminución del crecimiento económico, que se da como resultado de la inexistencia de una población que permita sostener el desarrollo rural del municipio de Chinavita. Así, se encuentra que la implicación está dada como un círculo vicioso, ya que, si no hay población económicamente activa no habrá mano de obra que trabaje la tierra, lo que impedirá que tanto las familias como el municipio tengan mayores ingresos, situación que derivará en que cada vez sea más difícil contar con el capital económico para contar con los insumos que se necesitan para trabajar la tierra y que puedan destinarse al ahorro y posterior inversión. Esto termina limitando e incluso sometiendo a la pobreza e incapacidad de lograr mayores niveles de desarrollo económico del municipio, escenario que motivará la migración hacia otros territorios y que terminará profundizando este contexto. Además de la pérdida del relevo generacional que se perderá: mano de obra, conocimiento y herencia de la tierra, entre otros factores.

\section{Pérdida de productividad}

En esta misma línea, la Cepal (2004), ratifica que el envejecimiento tiene importantes repercusiones en el desarrollo de los países y por la amplia variedad de sectores a los que afecta, siendo el sector productivo uno de los más afectados. El rápido envejecimiento demográfico tiene impactos económicos tanto en el nivel agregado como en el individual, siendo la productividad agregada del territorio la gran afectada.

Las entrevistas realizadas vuelven a señalar que la productividad es otra de las variables afectadas 
directamente por el envejecimiento, fenómeno que se asocia a la pérdida de productividad en sus cultivos tradicionales. En el grupo focal se pudo corroborar que la mano de obra de Chinavita se ha ido perdiendo, situación que ha impedido a los dueños de la tierra trabajarla y ponerla a producir, lo que, a su vez, ha impactado negativamente la productividad agregada de Chinavita. Adicionalmente, las actividades productivas propias del campo y la agricultura se hacen más exigentes en términos de tiempo y esfuerzo, lo que termina impactando negativamente la productividad de la economía del municipio, ya que, el esfuerzo físico y el tiempo necesario para cumplir con estos deberes diarios de la economía del territorio, que depende en gran parte de la agricultura, no disponen de mano de obra adicional que permita sustituir las horas/ hombre. Según los campesinos entrevistados, el total de la producción ha disminuido, hasta el punto que la mayoría cultivan para autoconsumo de ellos y sus familias (incluso las que viven en Bogotá). Igualmente, es normal la compra de una buena porción de los alimentos que se consumen. Es común sentir que se produce a pérdida, especialmente por la sobreoferta de productos que disminuye el precio de comercialización. A lo que se suma la pérdida parcial o total de las cosechas por el cambio climático y/o plagas.

La institucionalidad local también confirma el impacto que se identifica sobre la productividad. De acuerdo con este importante actor de Chinavita, en cabeza de Epsagro, ante la pérdida de población que permita explotar la tierra productivamente, se presenta una pérdida de la mano de obra para trabajar en el sector rural que cada día es más difícil conseguir y se hace más costosa. Epsagro señala que la capacidad de trabajo de las personas se ve disminuida con el proceso natural de envejecimiento, que hace que las personas pierdan capacidad física para trabajar y el deterioro de su salud, lo que también contribuye con esta realidad. Así, los adultos mayores no están en condiciones de aumentar o conservar su productividad, situación que sí podría surtir una población más joven.

Adicionalmente, el tamaño de la propiedad, sumado a los bajos niveles de tecnificación, impide una producción a una escala que genere excedentes para la comercialización, y, por ende, de utilidad monetaria al campesino. Sin embargo, culturalmente existe una resistencia hacia los procesos asociativos que se refleja en la existencia de solo cinco asociaciones productivas en el municipio: ganaderos, discapacitados porcicultores, cafeteros y mujeres campesinas. De estas, solo las dos últimas funcionan en la práctica, de acuerdo con lo expresado por Epsagro durante la entrevista. A esto se suma que no existen programas integrales de asistencia institucional que no solo promuevan el incremento en los niveles de producción, sino que, por el contrario, garanticen la generación de valor agregado y la comercialización del producto directamente al consumidor final, eliminando al intermediario, quien hasta el momento se queda con la mayor utilidad.

En este sentido, Cecilia López Montaño señaló que: "el envejecimiento es un proceso natural de la vida, pero cuando alcanza niveles críticos, impacta directamente sobre la productividad de los territorios, y esto a su vez sobre el desarrollo rural, ya que, la agricultura como fuente de ingresos de la ruralidad, está dada en términos de esta productividad que se pierde como producto de no contar con población que sustente el proceso y unos niveles de modernización de la industria que sean acordes a las nuevas exigencias de los mercados". Este detrimento de la productividad se ve profundizado por la falta de apoyo institucional para fortalecer la modernización y tecnificación del campo, lo que en el largo plazo condenará la economía del territorio a ser poco competitiva y a ser incapaz de cumplir las demandas del municipio.

Teniendo en cuenta que los principales productos de la economía y la agricultura de Chinavita son 
el maíz y el trigo, se estudió su comportamiento en cuanto a producción departamental, según datos de la Federación Nacional de Cultivadores de Cereales y Leguminosas, Fenalce, (2016). En cuanto al maíz no hubo ningún hallazgo, pero en relación al trigo se encontró que la productividad efectivamente había disminuido. Los campesinos expresaron, de común acuerdo, que su producción de cultivos tradicionales se ha visto reducida significativamente, como resultado de no contar con mano de obra que les permita sostener los cultivos que históricamente tuvieron en décadas pasadas; adicionalmente, la transmisión de conocimiento de estos cultivos se dificulta por la falta del relevo generacional.

En este sentido, se encuentra que el envejecimiento trae consigo una pérdida de productividad que afecta directamente la competitividad de los territorios cuando alcanza niveles críticos. Este hallazgo, al igual que el relacionado con el crecimiento económico, se fundamenta en la disminución de mano de obra económicamente activa que sea capaz de mantener la capacidad de trabajar la tierra a altos niveles de productividad.

\section{Conclusiones}

Esta investigación tuvo como objetivo el determinar las implicaciones del envejecimiento sobre el desarrollo rural de Boyacá. A partir del estudio de caso del municipio de Chinavita, se pudo determinar que este departamento ha atravesado por procesos migratorios, como consecuencia directa de los modelos de desarrollo y del enfoque de las políticas públicas implementadas en su territorio. Esto ha derivado en una situación de envejecimiento marcado de su población, lo que a su vez se refleja el la reducción de la economía del territorio y en la disminución del crecimiento económico y la pérdida de productividad.

En cuanto al envejecimiento, este concepto se puede analizar desde diferentes perspectivas: en relación con la dimensión biológica y su vínculo con el envejecimiento demográfico; el envejecimiento activo; y los derechos humanos y el sistema de protección social integral. El concepto de vejez se relaciona con un deterioro progresivo y generalizado de las funciones del cuerpo y mente del ser humano, en razón a los cambios biológicos y naturales que surgen con la edad. Además, el proceso de envejecimiento presenta una influencia directa por factores del entorno como: los hábitos de vida y las enfermedades, entre otros. Lo anterior indica la complejidad del concepto y la forma en que este debe abordarse en la formulación de políticas públicas sobre el tema.

La relación entre el envejecimiento y el desarrollo rural, indica que el envejecimiento proyecta desafíos sociales, económicos y humanitarios. Entre ellos sobresalen las afectaciones del crecimiento económico, la disminución de población económicamente activa que sea capaz de mantener las estructuras productivas de las economías locales, los regímenes de jubilación y salud que pueden verse seriamente afectados en su sostenibilidad financiera. Igualmente, se altera la continuidad en el largo plazo de programas sociales que atienden necesidades básicas (alimentación, vestimenta y vivienda), alta incidencia de la pobreza que persiste y profundiza la inequidad social, una probable tendencia hacia el deterioro de las estructuras familiares de apoyo al adulto mayor, y relaciones de dependencia y perpetuidad con fuertes implicaciones de las finanzas públicas.

Lo anterior está en relación directa con los procesos migratorios de la población más joven, quienes salen del campo motivados por la búsqueda de mejores oportunidades de empleo y educación que evidencian la ausencia de la institucionalidad pública en el sector rural. Esto produce un efecto de pérdida de identidad; es decir, la población más joven se ve presionada a abandonar su hogar, dejando el campo, migrando hacia la ciudad en busca de mejores oportunidades de educación y empleo, que le permitan un mayor crecimiento personal expresado en un aumento de sus 
ingresos acompañado de menores esfuerzos laborales. Esta reducción en la población joven que habita en zonas rurales, influye gravemente no solo en el envejecimiento del campo, también en los niveles de producción y productividad del mismo, incidiendo negativamente en ellos.

A la ausencia de mano de obra se suman los altos costos de producción, que reduce cada vez más la competitividad del sector. Como resultado, muchas de las familias campesinas cultivan mayormente para el autoconsumo, lo que disminuye el nivel de ingresos económicos, que apenas alcanzarían para el cubrimiento de las necesidades básicas.

En estecontexto, la investigación pone en evidencia la necesidad de promover una política pública de desarrollo rural, que haga frente a los procesos de envejecimiento del sector, desde el análisis de sus causas y consecuencias. Es necesario pensar en lo rural como la garantía a la soberanía alimentaria del país, lo que permitirá a su vez, el rescate de prácticas y valores tradicionales campesinos. En esta medida, futuras investigaciones pueden estar orientadas en determinar el papel de los adultos mayores en los procesos de desarrollo local pensados desde el territorio. Igualmente, pueden ser valiosos aquellos estudios que evidencien las condiciones necesarias para que los jóvenes decidan realizar un proyecto de vida en el campo. Para ello, puede ser importante el desarrollo de estudios cuantitativos que validen los resultados de la presente investigación, principalmente frente a los procesos migratorios, la afectación en el crecimiento económico y la sostenibilidad financiera del sistema de seguridad y protección social al adulto mayor.

\section{Referencias}

Alcaldía de Chinavita (2016). Plan de desarrollo: Unidos por Chinavita 2016-2019. Recuperado de: http://www.chinavita-boyaca.gov.co/index. shtml?x=3013256
Andrews, G. R. (2000). Los desafíos del proceso de envejecimiento en las sociedades de hoy y del futuro. Encuentro latinoamericano y caribeño sobre las personas de edad: ponencias presentadas al seminario técnico. Santiago de Chile, Chile.

Comisión Económica para América Latina y el Caribe, CEPAL. (1990). Transformación productiva con equidad. La tarea prioritaria de América Latina y el Caribe en los años noventa. Chile.

Comisión Económica para América Latina y el Caribe, CEPAL (2000). Los desafíos del proceso de envejecimiento en las sociedades de hoy y del futuro. Santiago de Chile, Chile.

Comisión Económica para América Latina y el Caribe, CEPAL. (2004). Población, envejecimiento y desarrollo. San Juan, Puerto Rico.

Departamento Administrativo Nacional de Estadística, DANE. (2005). Censo 2005. Bogotá, Colombia.

Departamento Administrativo Nacional de Estadística, DANE. (2010). Estudios postcensales: proyecciones nacional y departamentales de población 2005-2020. Bogotá, Colombia.

Departamento Nacional de Planeación, DNP. (2015). Misión para la Transformación del Campo. Bogotá, Colombia.

Federación Nacional de Cultivadores de Cereales y Leguminosas, Fenalce. (2016). Departamento económico y apoyo a la comercialización: indicadores cerealistas 2016. Recuperado de: http:// www.agronomia.unal.edu.co/sites/default/files/ IMGS/Indicador\%20Cerealista\%202016B.pdf

Fedesarrolloy Fundación Saldarriaga (2015). Misión Colombia envejece: cifras, retos y recomendaciones. Bogotá, Colombia: Fundación Saldarriaga Concha. 
Kay, C. (2007). Enfoques sobre desarrollo rural en América Latina y Europa desde mediados del siglo $X X$. La enseñanza del desarrollo rural: enfoques y perspectivas. Bogotá, Colombia: Javegraf.

López-Posada, J. C., \& Pachón-Ariza, F. A. (2017). Identificación de ventajas y desventajas de los canales de comercialización en las economías campesinas de dos municipios de Meta y Cundinamarca, Colombia. Revista de Investigación, Desarrollo e Innovación, 8 (1), 35-47. doi: 10.19053/20278306.v8.n1.2017.7369

Meuser, M., \& Nagel, U. (1991). ExpertInneninterviews - vielfach erprobt, wenig bedacht : ein Beitrag zur qualitativen Methodendiskussion. In: Garz, Detlef (Ed.); Kraimer, Klaus (Ed.): Qualitativ-empirische Sozialforschung : Konzepte, Methoden, Analysen. Wiesbaden, Alemania: Springer.

Misión para la Transformación del Campo, MTC. (2015). La Protección Social de la Población Rural. Bogotá, Colombia.

Organización Mundial de la Salud, OMS. (2015). Envejecimiento y Salud: Nota descriptiva No. 404.
Recuperado de: http://www.who.int/mediacentre/ factsheets/fs404/es/

Pérez-Correa, E., \& Farah-Quijano, M. (2002). Los modelos de desarrollo y las funciones del medio rural en Colombia. Cuadernos de desarrollo Rural, 49. Recuperado de: http://revistas.javeriana. edu.co/index.php/desarrolloRural/article/ viewFile/1987/1268

Sampieri, H., Fernández, R. C., \& Lucio, B. (2006). Metodología de la Investigación. México: McGrawHill Interamericana.

Sierra-Roberto, J. (2012). Perspectivas y escenarios de desarrollo rural para las comunidades campesinas: estudio de caso Municipio de Sora, Boyacá, Colombia. Revista de Investigación, Desarrollo e Innovación, 2 (2), 8-17. Recuperado de http://revistas.uptc.edu.co/index.php/ investigacion_duitama/article/view/1311/1306

Stake, R. (1995). The art of Case Study Research. California, USA: Sage.

Vasilachis-de Gialdino, I. (2006). Estrategias de Investigación Cualitativa. Barcelona, España: Gedisa. 